\title{
PHYSICAL CHEMISTRY OF SOLUTIONS
}

\section{Coordination Numbers of Bivalent Ions in Organic Solvents}

\author{
M. A. Orekhov ${ }^{a, b, c, *}$ \\ ${ }^{a}$ Joint Institute for High Temperatures, Russian Academy of Sciences, Moscow, 125412 Russia \\ ${ }^{b}$ Moscow Institute of Physics and Technology (State University), Moscow, 141701 Russia \\ ${ }^{c}$ National Research University, Higher School of Economics, Moscow, 123458 Russia \\ *e-mail:mo2411@mail.ru \\ Received August 27, 2020; revised January 21, 2021; accepted March 12, 2021
}

\begin{abstract}
Molecular dynamic models are created for properties of bivalent ions in organic solvents. It is shown that molecules of the considered solvents bound to ions via oxygen atoms. A theoretical model is developed that describes the ion coordination number. The coordination number in this model is determined by the ratio between the sizes of the ion and the atom organic molecule bound to it. It is shown that the coordination number depends weakly on the solvent and strongly on the type of ion. A value of $0.13 \mathrm{~nm}$ is obtained for the effective size of an oxygen atom bound to a bivalent ion. The constructed theoretical model agrees with the results from molecular dynamic calculations and the available experimental data.
\end{abstract}

Keywords: organic solvents, ion solvation, molecular dynamics, atomic radii

DOI: $10.1134 / \mathrm{S} 0036024421100204$

\section{INTRODUCTION}

Properties of ions in liquids are vital for describing different electrochemical processes [1]. The formation of solvation shells around ions has a crucial effect on such processes. Ion solvation affects charge transfer [2, 3], interaction with surfaces [4], and the kinetics of chemical interactions [5]. The properties of the solvation shells in solutions based on organic solvents are especially critical in the development of batteries [6-11].

Properties of the solvation shells of atomic ions in aqueous solutions have been described comprehensively in both theory and practice. Values of coordination numbers [12], shell lifetimes [13], and energies of solvation [14] have been determined in particular.

Properties of lithium ions have mainly been considered in theoretical and experimental studies on organic solvents $[5,15,16]$, due to the focus on developing materials for lithium ion batteries. In addition to lithium ion batteries, technologies based on bivalent ions are now being developed. The use of magnesium [17], calcium [18], and other ions [19] is actively considered. The list of promising solvents is constantly growing as well [19]. This results in a great many ionsolvent combinations on whose basis new-generation batteries can be created. There is thus a need to create a universal description of the properties of solvation shells in a wide range of organic solvents (including the shells of bivalent ions).

The main characteristic of solvation shell geometry is the ion coordination number (i.e., the number of molecules in the immediate neighborhood of an ion). In this work, we deal with the solvation shells and coordination numbers of bivalent ions in the six solvents most often considered in the development of batteries $[20,21]$. Numerical calculations are made by means of molecular dynamics [22], which are used to model the properties of materials [23-25]. A theoretical model describing the coordination numbers of ions is constructed. To confirm the completeness of our theoretical conclusions, beryllium ions (which are not used in batteries) are considered in addition to calcium and magnesium ions.

In the first section, we describe molecular dynamic modeling and the potentials of interatomic interaction that are used. In the second section, we present data from the molecular dynamic calculations and compare them to literature data. In the third section, we present a model that allows us to determine the ion coordination number in a liquid without molecular dynamic calculations. It is shown that this model agrees with both calculated and experimental values.

\section{COMPUTATIONAL PROCEDURE}

We considered the solvents dimethyl carbonate (DMC), diethyl carbonate (DEC), ethyl methyl carbonate (EMC), ethylene carbonate (EC), ethyl acetate (EA), and methyl acetate (MA) [20, 21].

The GROMOS force field was used [26] to describe the potential of interaction between solvent atoms, along with the interaction between ions $\mathrm{Mg}^{2+}$ and $\mathrm{Ca}^{2+}$ and the atoms of solvent molecules. There is 


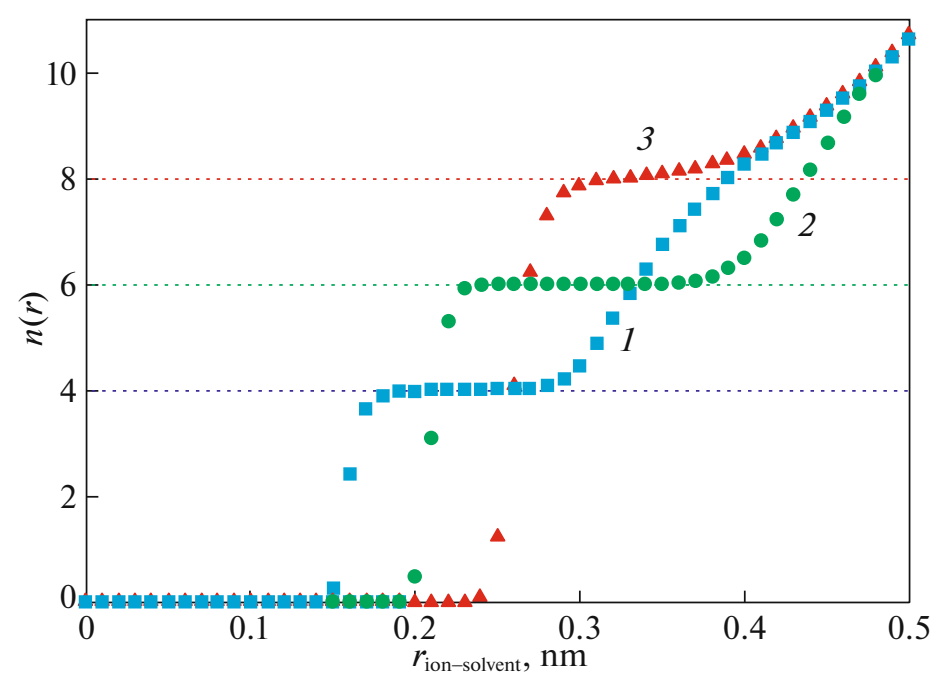

Fig. 1. Dependences of the average number of molecules on the distance to an ion in dimethyl carbonate. Blue squares (1) denote data for $\mathrm{Be}^{2+}$ ions; green dots (2), the data for $\mathrm{Mg}^{2+}$ ions; red triangles (3), data for $\mathrm{Ca}^{2+}$ ions.

no parametrization for $\mathrm{Be}^{2+}$ ions in this force field. Interaction between these ions and the atoms of liquids is presented in the form of the Coulomb and Lennard-Jones potentials:

$$
U(r)=4 \varepsilon\left[(\sigma / r)^{12}-(\sigma / r)^{6}\right] .
$$

Parameters of potential (1) from publication [27] were taken to define the interaction of $\mathrm{Be}^{2+}$ with atoms of a liquid. The parameters of interaction among oxygen atoms for calculations with this ion were also taken from [27]. The topology of the bonds between atoms in the solvent molecules and partial charges on these atoms were taken from the Automated Topology Builder (ATB) database [28, 29]. Long-range interaction was described using the particle mesh Ewald (PME) approach [30].

Classical molecular dynamics was used in our calculations. The system temperature was $330 \mathrm{~K}$. The pressure was 1 bar. The numerical integration step was $1 \mathrm{fs}$. The number of solvent molecules was 300 . A check of the dependence of the coordination number on the number of solvent molecules for diethyl carbonate revealed that deviations from the results relative to a system consisting of 1200 molecules were no greater than $2 \%$. Molecules were added to random places of the computational mesh. Then one ion was added to a random place. The energy was minimized to preclude overlap of molecules. The system was then brought to equilibrium within 450 ps in calculations using a thermostat and a barostat. Finally, the coordination number was calculated in $1.55 \mathrm{~ns}$. The results were averaged over time and 16 trajectories with independent initial conditions. The calculations were made using the GROMACS software toolkit [31].

The number of ion neighbors at distance $r$ was calculated as the number of molecules with at least one atom located from the ion at a distance smaller than $r$. The coordination numbers were obtained from the horizontal pieces of the plot of the number of neighbors on $r$. An example of such a plot is shown in Fig. 1.

Fluctuations of the coordination numbers were calculated from the differences of their values $\left(C_{i}\right)$ on $N=16$ trajectories with independent initial conditions. The formula

$$
\Delta C=\sqrt{\sum\left(C_{i}-\langle C\rangle\right) / N-1}
$$

was used.

\section{RESULTS FROM CALCULATIONS VS. EXPERIMENTAL VALUES \\ Results from Calculations}

The coordination numbers obtained via molecular dynamics are given in Fig. 2. The coordination numbers depend weakly on the choice of solvent. Changes in the coordination numbers for specific ions are no greater than 0.1 when different solvents are used for all substances. Fluctuations in the coordination numbers were calculated in addition to average values. They are illustrated in Fig. 2 in the form of error crosses. The fluctuations surpass the deviations from the mean coordination number for specific ions of all substances.

The weak dependence of the coordination number on the type of solvent can be explained by the molecules of the considered solvents being bound to the ions through oxygen atoms. To verify this, we plotted the dependences of the number of atoms in a solvent molecule on the distance to an ion. An example of such a dependence is shown in Fig. 3 for a $\mathrm{Be}^{2+}$ ion in dimethyl carbonate (DMC). It is seen that four oxygen atoms are the ones nearest the ion. On the other hand, 


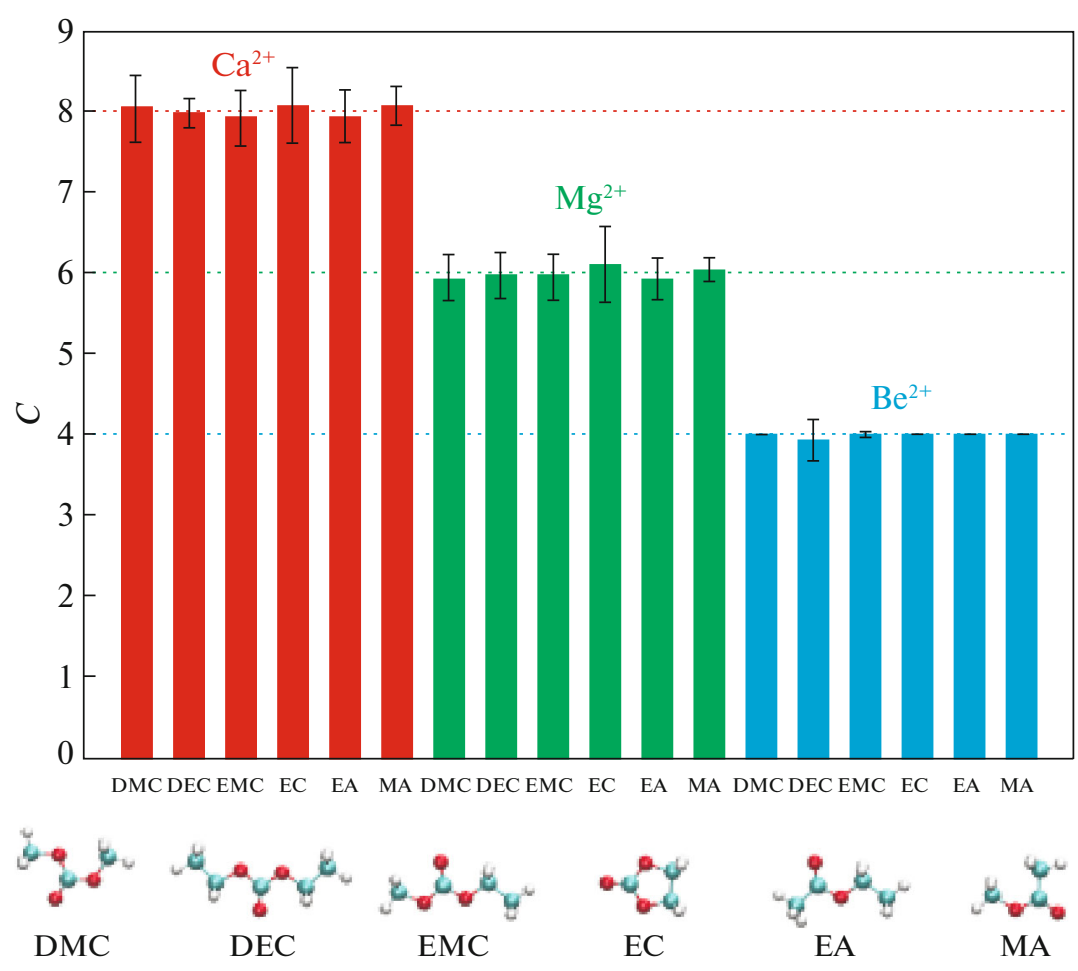

Fig. 2. Coordination numbers of ions in different organic solvents, according to molecular dynamics data. The three groups of columns with different colors correspond to different ions $\left(\mathrm{Ca}^{2+}, \mathrm{Mg}^{2+}\right.$, and $\left.\mathrm{Be}^{2+}\right)$. Abbreviated names of the corresponding solvents are given at the bottom. The error crosses correspond to fluctuations in the coordination numbers. The statistical error of calculations is much lower than the value of fluctuations.

it is clear from Fig. 1 that the ion is surrounded by four molecules. The oxygen atoms surrounding the ion therefore belong to different molecules. At the same time, other carbon and oxygen atoms of the molecule are much farther from the ion. A similar picture is observed for other solvents and ions, confirming the hypothesis of ion bonding through oxygen atoms. An ion is thus surrounded by a shell of oxygen atoms, with one atom from each neighboring molecule.

There is a strong dependence of the coordination number on the type of ion (Fig. 2). The change in the coordination number upon moving from ion to another is greater than the root-mean-square fluctuation.

\section{Comparison to Experiments}

All of the considered solvents have a common feature: an ion is bound to a solvent molecule through the latter's oxygen atom. This is analogous to ions in water, where the ion is also surrounded by oxygen atoms. Experimental values of the coordination numbers of ions in water are indicated as horizontal lines in Fig. 2. The experimental value of the coordination number in water coincides with that of the considered organic solvents for all our ions [12, 27]. In the literature, there is some spread of the experimental data for calcium ions [32], from 7.2 to 10 . A value of 8 was obtained in most experiments [27, 32, 33].

There is thus agreement with the experimental values of the coordination numbers of ions in water. Another analogy is the comparison to the coordination numbers in metal oxide crystals. The values of the coordination numbers of the corresponding ions in organic solvents coincide with those of the crystals of oxides of these metals [33].

\section{THEORETICAL MODEL OF COORDINATION NUMBERS}

The Magnus-Goldschmidt model can be adapted to theoretically describe the coordination numbers of ions in a liquid [34, 35]. This model was built to describe the coordination numbers in crystals. It is based on the assumption that the coordination number depends on the ratio between the radii of neighboring ions. Atoms of the crystal are described as solid spheres, and a stable configuration of the ionic surroundings is determined from geometric considerations.

To describe the coordination number of an ion in a liquid, we must use the radius of an organic molecule's atom bound to an ion instead of the radius of an atom in a crystal. Oxygen atoms are bound to ions in the considered solvents. This results in the formula 


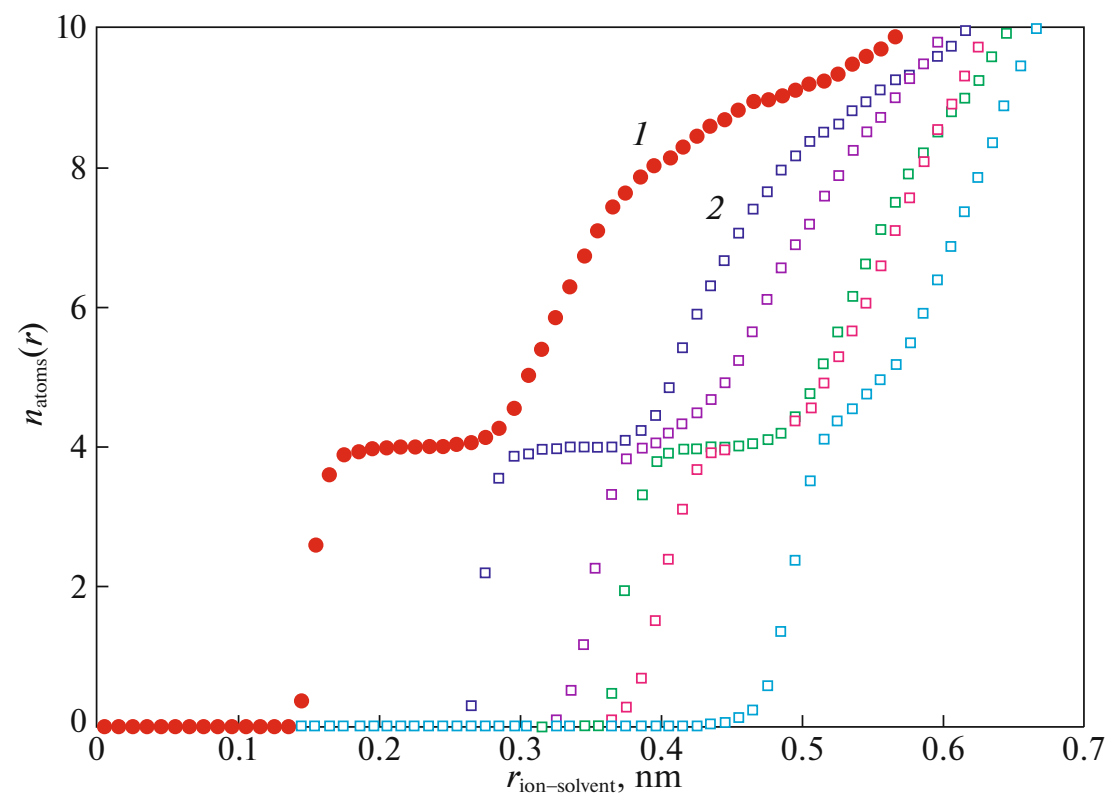

Fig. 3. Dependences of the numbers of different types of atoms on the distance to $\mathrm{Be}^{2+}$ ions in dimethyl carbonate. Red dots ( 1 ) indicate data for oxygen bound to ions. Squares of various colors (2) indicate data for different carbon atoms and other oxygen atoms of the dimethyl carbonate molecule. Data for hydrogen atoms are not shown.

$$
C=\left\{\begin{array}{lll}
8, & r_{\text {ion }} / r_{\text {oxygen }} & \in[1 ; 0.732] \\
7, & r_{\text {ion }} / r_{\text {oxygen }} \in[0.592 ; 0.732] \\
6, & r_{\text {ion }} / r_{\text {oxygen }} \in[0.414 ; 0.592] \\
4, & r_{\text {ion }} / r_{\text {oxygen }} \in[0.225 ; 0.414] \\
3, & r_{\text {ion }} / r_{\text {oxygen }} \in[0.155 ; 0.225],
\end{array}\right.
$$

where $C$ is the ion coordination number, $r_{\text {ion }}$ is the ionic radius, and $r_{\text {oxygen }}$ is the radius of the oxygen atom nearest to an ion.

To use this model, we need the values of the radii of the metal ion and the organic molecule oxygen atom. There are different definitions of atomic and ionic radii. In one approach, we use interatomic distances in different substances containing such atoms and ions. It is presumed that the distance between a pair of atoms is the sum of their radii. We therefore can obtain the radius of one atom by specifying the radius of the other. Assuming that we know the radius of an oxygen atom and the ion-atom distance, we obtain

$$
r_{\text {ion }} / r_{\text {oxygen }}=\left(d_{\text {ion-oxygen }}-r_{\text {oxygen }}\right) / r_{\text {oxygen }}
$$

for the ratio between the radii of oxygen and the ion. Here, $d_{\text {ion-oxygen }}$ is the average distance between an ion and an oxygen atom in a substance. By specifying the $r_{\text {oxygen }}$ value, we define that of $r_{\text {ion }}$ and obtain the one for the coordination number using formula (2). The $r_{\text {oxygen }}$ value can be obtained from the distance between oxygen atoms in different substances. This choice will be ambiguous and depend on the type of the substance. Estimates of the radius of an oxygen atom vary from 0.13 to $0.15 \mathrm{~nm}[34,36]$. The precision of speci- fying the oxygen radius is vital for such small ions as $\mathrm{Be}^{2+}$. The $d_{\text {ion-oxygen }}$ value is close to $r_{\text {oxygen }}$ for such ions, so the numerator of formula (3) changes greatly when a change in $r_{\text {oxygen }}$ is small. This results in different estimates of the coordination number, due to errors in the $r_{\text {oxygen }}$ values.

For the most accurate description of the properties of ions in organic solvents, it is suitable to use $r_{\text {oxygen }}$ as a fitted parameter of the model. To determine the value of this parameter, we calculated the coordination numbers of ions with different sizes. The results from our molecular dynamic calculations are presented in Fig. 4. A wide range of ion sizes were chosen by varying the parameters of potential (1). The ionoxygen atom distance was determined as the first maximum of the pair correlation function. Agreement between the results from molecular dynamic calculations and the theoretical model was reached at $r_{\text {oxygen }}=0.13 \mathrm{~nm}$. Deviation of $r_{\text {oxygen }}$ by $0.1 \mathrm{~nm}$ results in a considerable discrepancy between the model and the molecular dynamics data.

On the other hand, using the experimental value of the distance between oxygen atoms in water yields an estimate of $r_{\text {oxygen }}=0.14 \mathrm{~nm}$ for the radius of oxygen [34]. Table 1 presents coordination numbers obtained using different oxygen radii.

The values obtained at $r_{\text {oxygen }}=0.14 \mathrm{~nm}$ agree with the calculated and experimental data for $\mathrm{Mg}^{2+}$ and $\mathrm{Ca}^{2+}$ ions. There is a discrepancy with the results for $\mathrm{Be}^{2+}$ ions. The values obtained from the interatomic distances in molecular dynamics agree with the theoretical and experimental ones for all ions at 


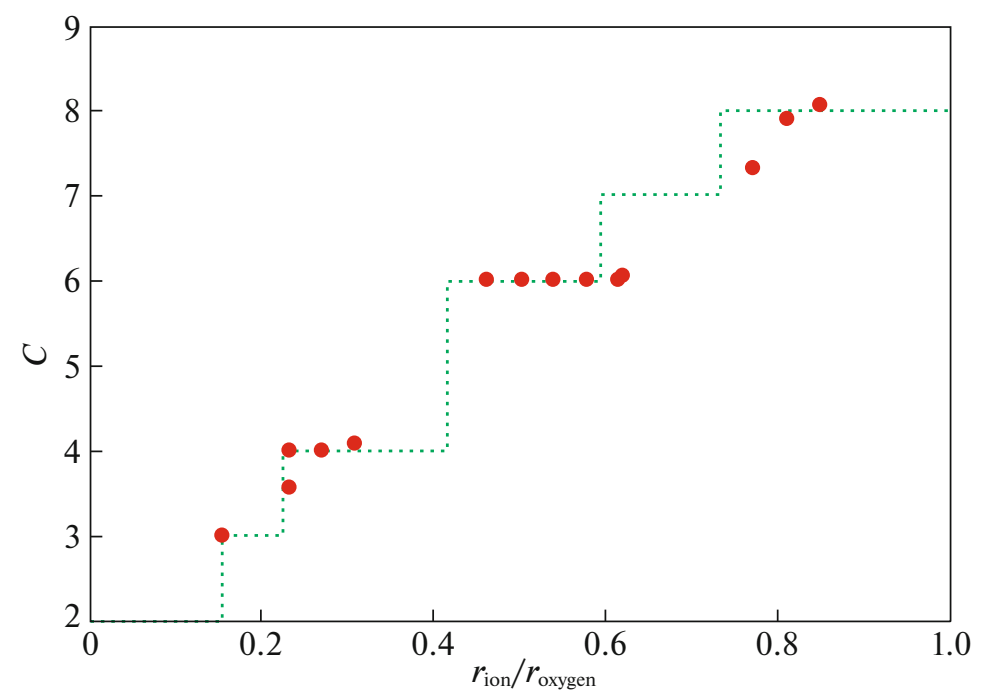

Fig. 4. Dots indicate the coordination numbers of ions with size $r_{\text {ion }}$ in dimethyl carbonate, according to the molecular dynamics data. The line shows theoretical estimates of the coordination numbers according to (2) the modified Magnus-Goldschmidt model and (3) with $r_{\text {oxygen }}=0.13 \mathrm{~nm}$.

$r_{\text {oxygen }}=0.13 \mathrm{~nm}$. Using $r_{\text {oxygen }}=0.13 \mathrm{~nm}$ therefore allows us to obtain agreement between the theoretical model, the calculated data, and experiments, in contrast to $r_{\text {oxygen }}=0.14 \mathrm{~nm}$ determined from the interatomic distances in water.

\section{CONCLUSIONS}

Properties of the solvation shells of bivalent ions in organic solvents were investigated by means of molecular dynamics and theoretical models. It was shown that the ion coordination number depends weakly on the solvent for molecules bound to ions through oxygen atoms. On the other hand, there is a strong dependence on the type of ion. The coordination numbers of the ions in such solvents coincide with the coordination numbers in water. This is because ions in water molecules are also bound with oxygen atoms. A theo- retical approach generalizing the Magnus-Goldschmidt model to the coordination numbers of bivalent ions in solutions was constructed. The resulting formula allowed us to calculate coordination numbers based on the sizes of ions and the atoms bound to them. It was shown that using the radius of an oxygen atom as a fitted parameter allows us to obtain agreement between molecular dynamics and theoretical models in a wide range of ionic sizes. The theoretical model of the coordination number is sensitive to the value of this parameter. Our value of $0.13 \mathrm{~nm}$ for the radius of an oxygen atom is close to half the distance between oxygen atoms in water $(0.14 \mathrm{~nm})$. However, using a value of $0.14 \mathrm{~nm}$ for the oxygen radius does not allow us to obtain agreement between the model with molecular dynamics and experiments. The theoretical model and the molecular dynamic calculations agree with the available experimental data on the coordination numbers of ions in aqueous solutions.

Table 1. Distances $(d, \mathrm{~nm})$ between ions and oxygen atoms in different substances

\begin{tabular}{|c|c|c|c|c|c|c|c|c|c|c|}
\hline \multirow{2}{*}{ Ion } & \multirow{2}{*}{$d_{\text {oxide }}$} & \multirow{2}{*}{$d_{\text {water }}$} & \multirow{2}{*}{$d_{\text {organic }}$} & $C_{\text {oxide }}$ & $C_{\text {water }}$ & $C_{\text {organic }}$ & $C_{\text {oxide }}$ & $C_{\text {water }}$ & $C_{\text {organic }}$ & \multirow{2}{*}{$C_{\text {exp }}$} \\
\hline & & & & \multicolumn{3}{|c|}{$r_{\text {oxygen }}=0.14 \mathrm{~nm}$} & \multicolumn{3}{|c|}{$r_{\text {oxygen }}=0.13 \mathrm{~nm}$} & \\
\hline $\mathrm{Be}^{2+}$ & 0.164 & 1.67 & 1.65 & 3 & 3 & 3 & 4 & 4 & 4 & 4 \\
\hline $\mathrm{Mg}^{2+}$ & 0.21 & 0.209 & 0.1995 & 6 & 6 & 6 & 7 & 7 & 6 & 6 \\
\hline $\mathrm{Ca}^{2+}$ & 0.243 & 0.246 & 0.25 & 8 & 8 & 8 & 8 & 8 & 8 & 8 \\
\hline
\end{tabular}

The experimental data for oxides $d_{\text {oxide }}$ and water $d_{\text {water }}$ are taken from [32]. The data for organic solvents $d_{\text {organic }}$ were found from the first peak of the pair correlation function obtained by molecular dynamics. The $d_{\text {organic }}$ values coincide for all of the solvents considered in this work. The theoretical values of the coordination numbers were calculated using (2) and (3) from the corresponding $d$ and $r_{\text {oxygen }}$ values. The $C_{\exp }$ values correspond to the experimental value [12, 27] of the coordination number in water and the calculated values for the organic solvents obtained via molecular dynamics. 


\section{ACKNOWLEDGMENTS}

Our calculations were made on the computer cluster at the Russian Academy of Sciences' Joint Supercomputer Center and the High-Performance Computing Center at the Joint Institute for High Temperatures.

The author is grateful to G.E. Norman for his helpful comments on the subject of our study.

\section{FUNDING}

This work was supported by the Russian Foundation for Basic Research, project no. 19-38-90063.

\section{OPEN ACCESS}

This article is licensed under a Creative Commons Attribution 4.0 International License, which permits use, sharing, adaptation, distribution and reproduction in any medium or format, as long as you give appropriate credit to the original author(s) and the source, provide a link to the Creative Commons license, and indicate if changes were made. The images or other third party material in this article are included in the article's Creative Commons license, unless indicated otherwise in a credit line to the material. If material is not included in the article's Creative Commons license and your intended use is not permitted by statutory regulation or exceeds the permitted use, you will need to obtain permission directly from the copyright holder. To view a copy of this license, visit http://creativecommons.org/licenses/by/4.0/.

\section{REFERENCES}

1. C. Lefrou, P. Fabry, and J. Poignet, Electrochemistry: The Basics, with Examples (Springer, 2012).

2. K. Møller, R. Rey, M. Masia, and J. Hynes, J. Chem. Phys. 122, 114508 (2005).

3. A. V. Lankin, G. E. Norman, and M. A. Orekhov, Russ. J. Phys. Chem. A 93, 1421 (2019). https://doi.org/10.1134/s003602441908017x

4. Y. Yamada, Y. Takazawa, K. Miyazaki, and T. Abe, J. Phys. Chem. C 114, 11680 (2010).

5. V. S. Smirnov and S. A. Kislenko, Chem. Phys. Chem. 20, 1960 (2019).

6. M. D. Vedenyapina, T. L. Kulova, Yu. O. Kudryashova, A. M. Skundin, O. R. Malyshev, and L. M. Glukhov, Russ. J. Phys. Chem. A 94, 1276 (2020). https://doi.org/10.1134/S0036024420060308

7. S. V. Pavlov and S. A. Kislenko, Phys. Chem. Chem. Phys. 18, 30830 (2016).

8. V. S. Smirnov and S. A. Kislenko, Chem. Phys. Chem 19, 75 (2017).

9. O. R. Rakhmanova and A. E. Galashev, Russ. J. Phys. Chem. A 91, 921 (2017).

https://doi.org/10.1134/S003602441705020X
10. A. F. Shestakov, A. V. Yudina, G. Z. Tulibaeva, Yu. M. Shul'ga, A. A. Ignatova, and O. V. Yarmolenko, Russ. J. Phys. Chem. A 91, 1444 (2017). https://doi.org/10.1134/S0036024417080301

11. F. Chen, R. Kerr, and M. Forsyth, J. Chem. Phys. 148, 193813 (2018).

12. H. Ohtaki, Chem. Rev. 93, 1157 (1993).

13. A. Putnis and C. V. Putnis, Phys. Chem. Chem. Phys. 16, 7772 (2014).

14. D. Jiao, K. King, A. Grossfield, et al., J. Phys. Chem. B 110, 18553 (2006).

15. S. Han, Sci. Rep. 9, 5555 (2019).

16. J. Soetens, C. Millot, and B. Maigret, J. Phys. Chem. A 102, 1055 (1998).

17. M. Salama, I. Shterenberg, H. Gizbar, et al., J. Phys. Chem. C 120, 19586 (2016).

18. A. Ponrouch, J. Bitenc, R. Dominko, et al., Energy Storage Mater. 20, 253 (2019).

19. L. Cheng, R. Assary, X. Qu, et al., J. Phys. Chem. Lett. 6, 283 (2015).

20. J. Xie and Y. Lu, Nat. Commun. 11, 2499 (2020).

21. B. Flamme, G. Garcia, M. Weil, et al., Green Chem. 19, 1828 (2017).

22. G. E. Norman and V. V. Stegailov, Math. Models Comput. Simul. 5, 305 (2013).

23. M. Orekhov, Phys. Chem. Chem. Phys. 19, 32398 (2017).

24. A. Antropov and V. Stegailov, J. Nucl. Mater. 553, 152110 (2020).

25. J. Dai, W. Zhang, C. Ren, and X. Guo, J. Mol. Liq., 114059 (2020).

26. N. Schmid, A. Eichenberger, and A. Choutko, Eur. Biophys. J. 40, 843 (2011).

27. Zh. Li, L. F. Song, P. Li, K. M. Merz, Jr., J. Chem. Theory Comput. 16, 4429 (2020).

28. A. K. Malde, L. Zuo, M. Breeze, et al., J. Chem. Theory. Comput. 7, 4026 (2011).

29. M. Stroet, B. Caron, K. Visscher, et al., J. Chem. Theory Comput. 14, 5834 (2018).

30. T. Darden, D. York, and L. Pedersen, J. Chem. Phys. 98, 10089 (1993).

31. B. Hess, C. Kutzner, D. van der Spoel, and E. Lindahl, J. Chem. Theory Comput. 4, 435 (2008).

32. T. Megyes, T. Gr'aosz, and T. Radnai, J. Phys. Chem. A 108, 7261 (2004).

33. X. Lei and B. Pan, J. Phys. Chem. A 114, 7595 (2010).

34. L. T. Bugaenko, S. M. Ryabykh, and A. L. Bugaenko, Mosc. Univ. Chem. Bull. 63, 303 (2008).

35. B. K. Vainshtein, Modern Crystallography, Vol. 2: Crystal Structure (Nauka, Moscow, 1979) [in Russian].

36. A. Bondi, J. Phys. Chem. 63, 441 (1964).

Translated by Z. Smirnova 